\title{
Underdiagnosed Distal Colonic Volvuli on MDCT Imaging
}

\author{
Mitesh Kumar*, Siddharth Saravanan Vijayakumar, Bhawna Dev \\ Dept of Radiology, Sri Ramachandra Medical College and Hospital, Chennai, India \\ Email: ${ }^{*}$ kumarmitesh@yahoo.com, sidsrvnn@gmail.com, bhawnadev@gmail.com
}

Received 8 January 2014; revised 5 February 2014; accepted 13 February 2014

Copyright (C) 2014 by authors and Scientific Research Publishing Inc.

This work is licensed under the Creative Commons Attribution International License (CC BY). http://creativecommons.org/licenses/by/4.0/

CC) (i) Open Access

\begin{abstract}
Volvuli affecting the distal colon, specifically the splenic flexure onwards is underdiagnosed pathologies with non-specific clinical features. Delay in diagnosing these pathologies can lead to significant morbidity and mortality in terms of ischemia and perforation of bowel. Multi detector computed tomography (MDCT) with intravenous contrast is an accurate and reliable imaging modality allowing prompt diagnosis and management. We present an overview of the radiological features found in acute presentations of different types of colonic volvuli.
\end{abstract}

\section{Keywords}

Splenic Flexure; Volvulus; Ileosigmoid Knot; Sigmoid Colon; Multi Detector Computed Tomography

\section{Introduction}

Pain in the left iliac fossa is a common clinical complaint encountered in an emergency setting. Initial radiological evaluation is usually accomplished using ultrasound screening, which can rule out common pathologies. Dilated bowel loops are also identifiable using ultrasound, however, the limitations of this modality lie in its inability to ascertain the exact pathology behind the dilated loops.

MDCT provides a definitive method to assess the cause, site and type of bowel dilatation and also evaluate secondary complications such as ischemia leading to gangrene and perforation.

We present here three different entities-Ileosigmoid knot, splenic flexure volvulus, and sigmoid volvulus, found causing obstruction, diagnosed on MDCT and confirmed intra operatively.

Splenic flexure volvulus, sigmoid volvulus and the ileosigmoid knot affect the distal colon, and may manifest non-specific clinical features, and therefore contrast enhanced MDCT plays a crucial role in their accurate diag-

${ }^{*}$ Corresponding author. 
nosis and timely management.

\section{Ileosigmoid Knot}

A fifty year old male patient presented with severe abdominal pain and distention, with no passage of feces or flatus for twenty four hours. The patient's vitals were stable and routine laboratory investigations were normal. Plain abdominal radiograph showed dilated large and small bowel loops with multiple air fluid levels in small bowel (Figure 1). MDCT of abdomen with intravenous contrast administration showed dilated sigmoid colon, with a "coffee-bean" appearance on coronal images (Figure 2). Ileal bowel loops were seen twisted around the base of the sigmoid colon, creating a "whirl" sign, causing closed loop obstruction of both sigmoid colon and ileal loops. Multiple air-fluid levels and small bowel fecal mottling were also observed. No signs of gangrene were seen (Figures 3 and 4).

Patient underwent surgery twelve hours after the CT study was performed owing to low hemoglobin count. Intra-operatively, there was a large dilated, gangrenous sigmoid colon with two complete turns of ileal loops at its base. The ileal loops were found to be viable (Figure 5). The gangrenous segment was resected and anastomosis was performed.

\section{Sigmoid Volvulus}

A fifty year old male patient was admitted with complaints of diffuse abdominal pain and distension, with recurrent bouts of vomiting. On examination he was found to have diffused abdominal tenderness with diminished bowel sounds. The MDCT scanogram revealed a dilated sigmoid colon with abrupt tapering giving it the "beak appearance" with accompanying dilated large bowel loops (Figure 6). Images obtained after administration of intravenous contrast showed a distended ahaustral sigmoid colon (Figure 7). The distended sigmoid colon loop was seen extending to the left hemi diaphragm (left flank overlap sign) and overlapping the lower border of liver shadow (liver overlap sign). The whirl sign was seen due to twisted sigmoid mesocolon and beak sign at the level of twist due to tapering of opposed loops (Figure 8). The apex of the sigmoid colon was seen above T10 level vertebra. The sigmoid colon was seen to lie cephalic to the transverse colon, an appearance referred to as the Northern exposure sign (Figure 6).

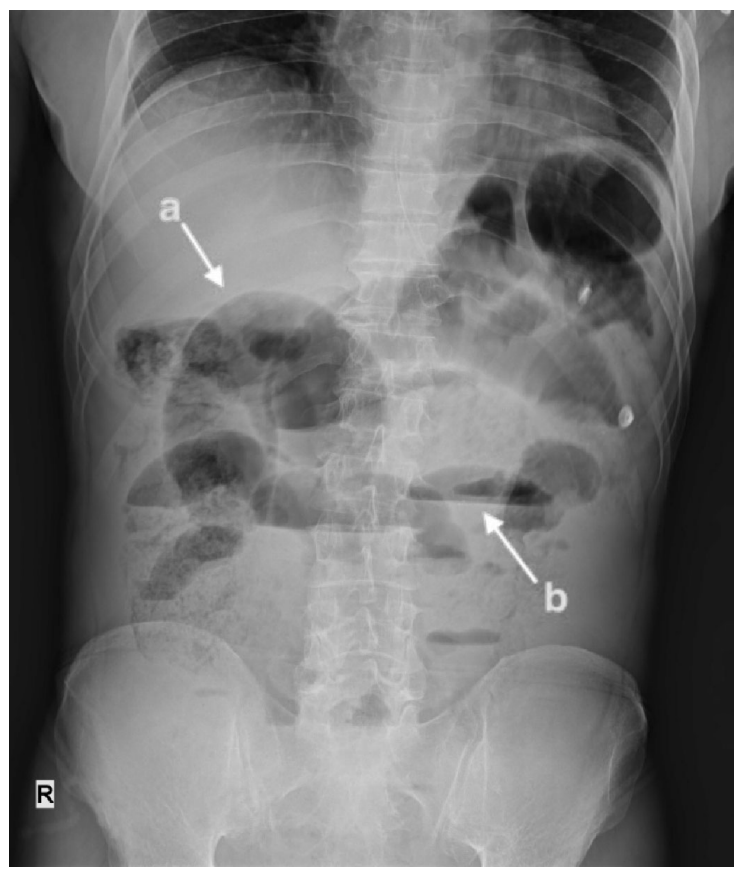

Figure 1. Plain abdominal radiograph demonstrating (a) dilated large bowel loop and (b) dilated small bowel loops with air-fluid levels. 


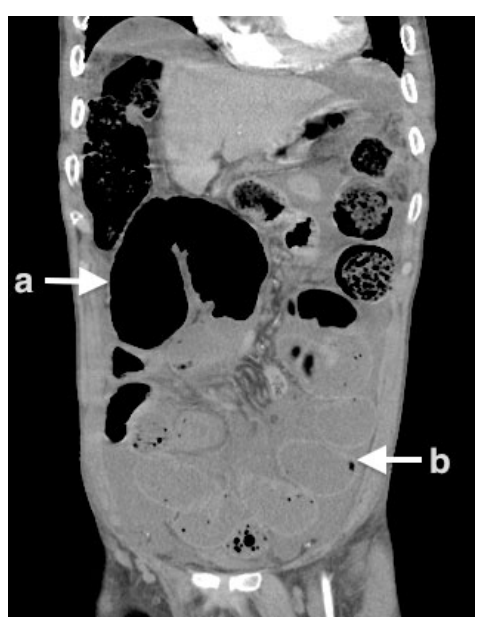

Figure 2. Coronal section of contrast enhanced MDCT scan showing (a) dilated large bowel loop and (b) dilated small bowel loops with airfluid levels (corresponding to plain radiograph in Figure 1).

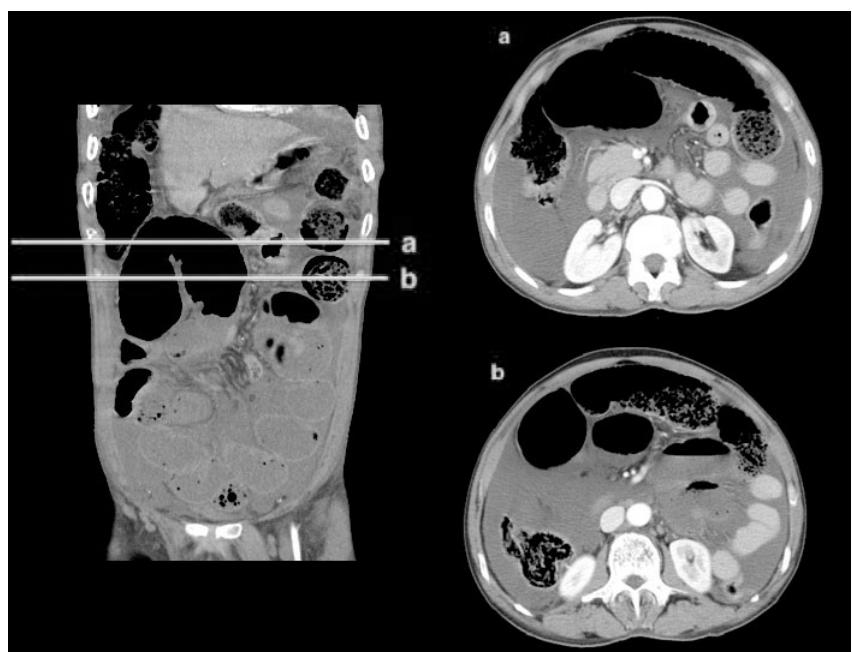

Figure 3. Axial sections (a) and (b) corresponding to levels shown in coronal image, showing closed loop sigmoid colon obstruction.

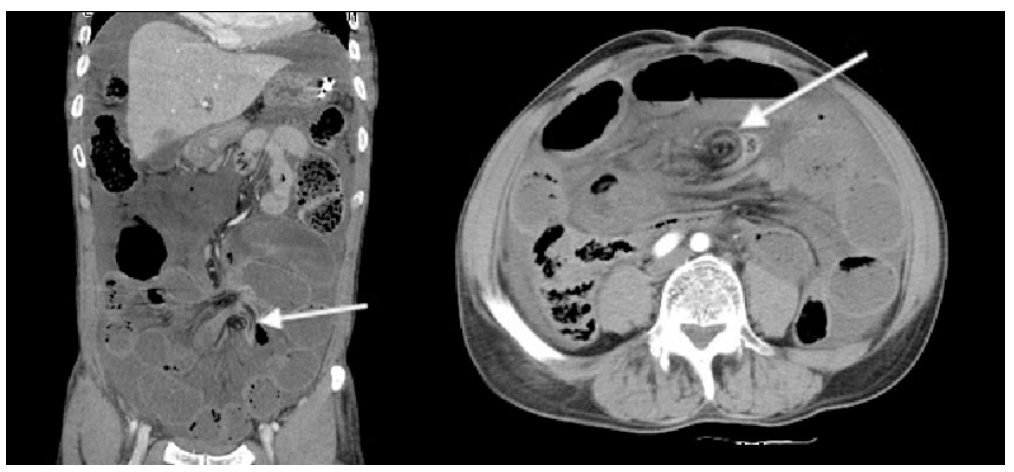

Figure 4. (a) Coronal and (b) axial sections of contrast enhanced CT showing whirling of ileal loops around sigmoid colon. 


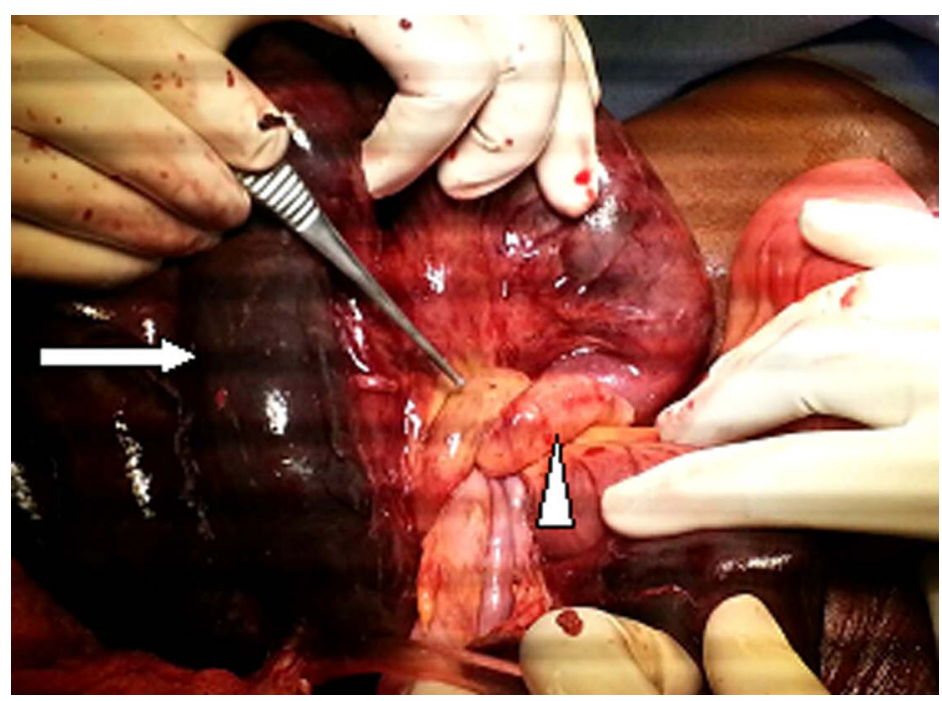

Figure 5. Intra-operative photograph showing dilated and gangrenous sigmoid colon (white arrow) with two ileal loops (white arrow head) twisting at its base.

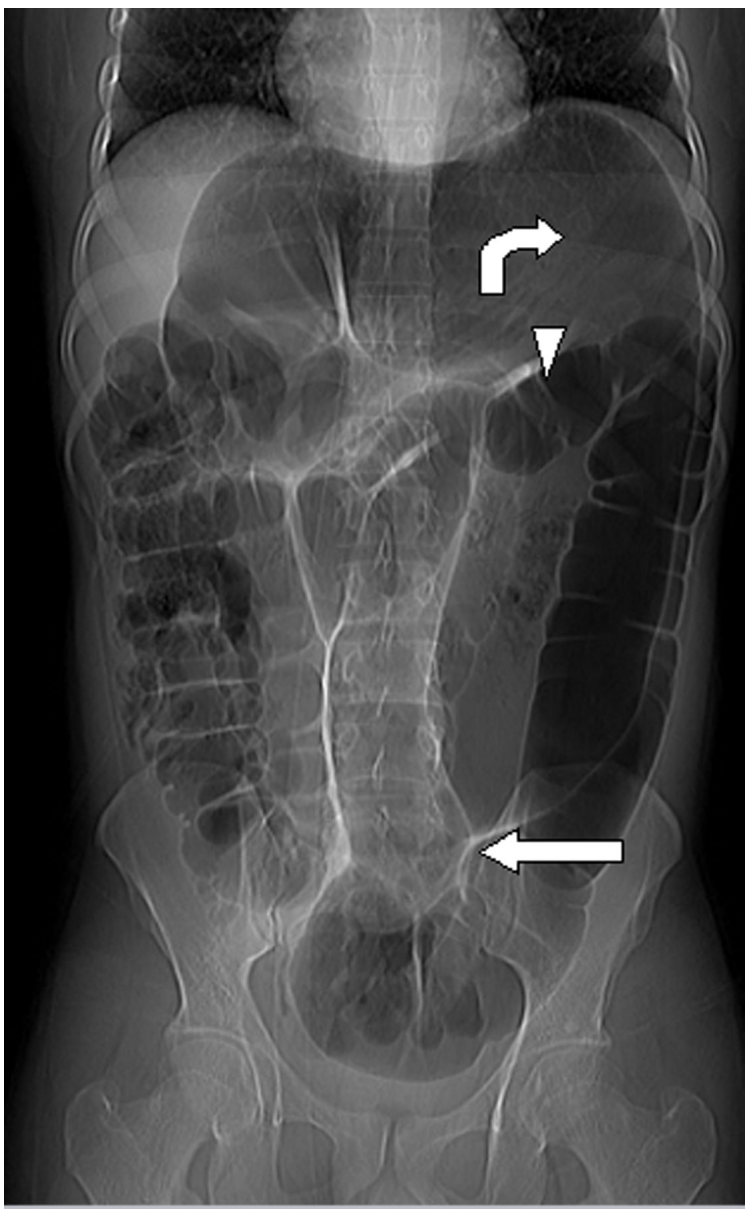

Figure 6. Scanogram of MDCT scan showing dilated sigmoid colon with beak appearance (white arrow), cephalic position of sigmoid colon (curved arrow) with respect to transverse colon (white arrow head). 


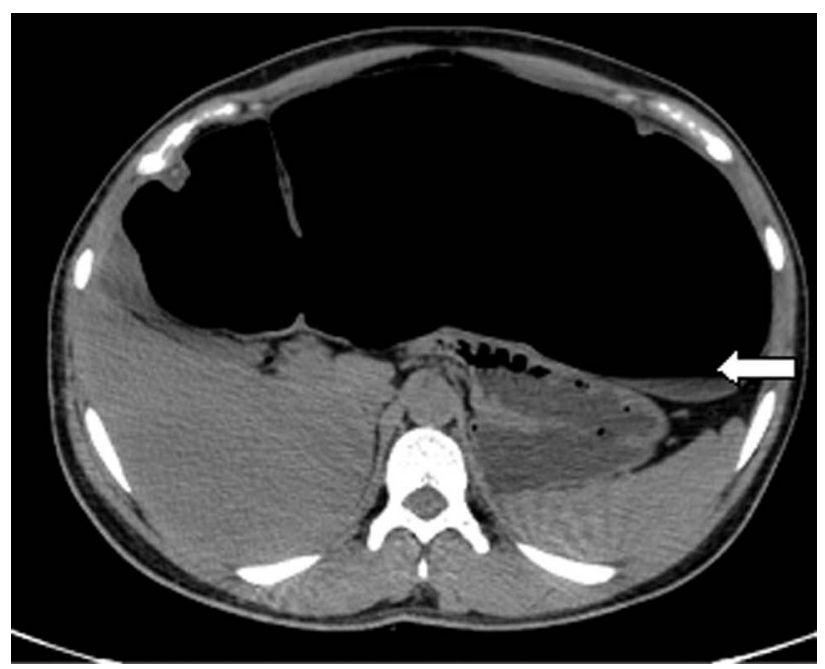

Figure 7. Axial plain MDCT scan of abdomen showing dilated ahaustral sigmoid colon (white arrow).

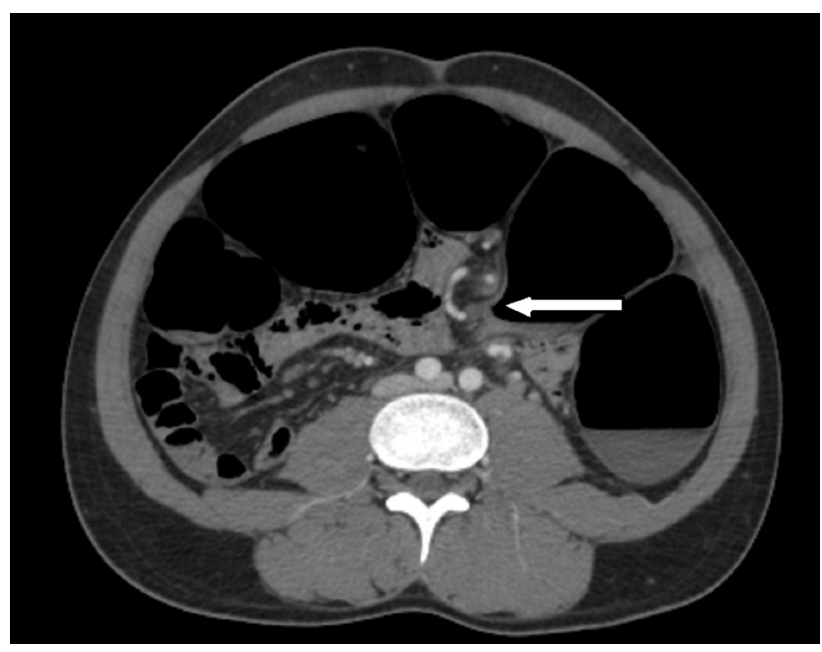

Figure 8. Axial contrast enhanced MDCT scan of abdomen showing whirl sign due to twisted sigmoid colon (white arrow).

Intra-operatively dilated, gangrenous sigmoid colon was seen twisted around its mesenteric root. Proximal colonic dilatation was noted (Figure 9). Resection and anastomosis was performed and the patient recovered uneventfully.

\section{Splenic Flexure Volvulus}

A twenty five year old male patient presented with acute abdominal pain, localized to the left flank. Patient also complained of abdominal distention with no passage of feces or flatus for over twenty four hours. On examination he was found to have tenderness and fullness over the left hypochondrium with decreased bowel sounds. Routine blood investigations were normal. Supine abdominal plain radiograph revealed dilated twisted large bowel loop in the region of splenic flexure with "coffee bean sign” and dilated proximal large bowel loops (Figure 10). The scanogram of MDCT showed the same findings as seen in plain supine abdominal radiograph (Figure 11). Plain scan of MDCT showed dilatation of the transverse and splenic flexure of colon (Figure 12). MDCT with intravenous contrast administration showed twisted splenic flexure around its mesentery, resulting in an inverted coffee bean appearance on coronal images (Figures 13(a) and 13(b)). No signs of gangrene or pneumoperitoneum were seen. 


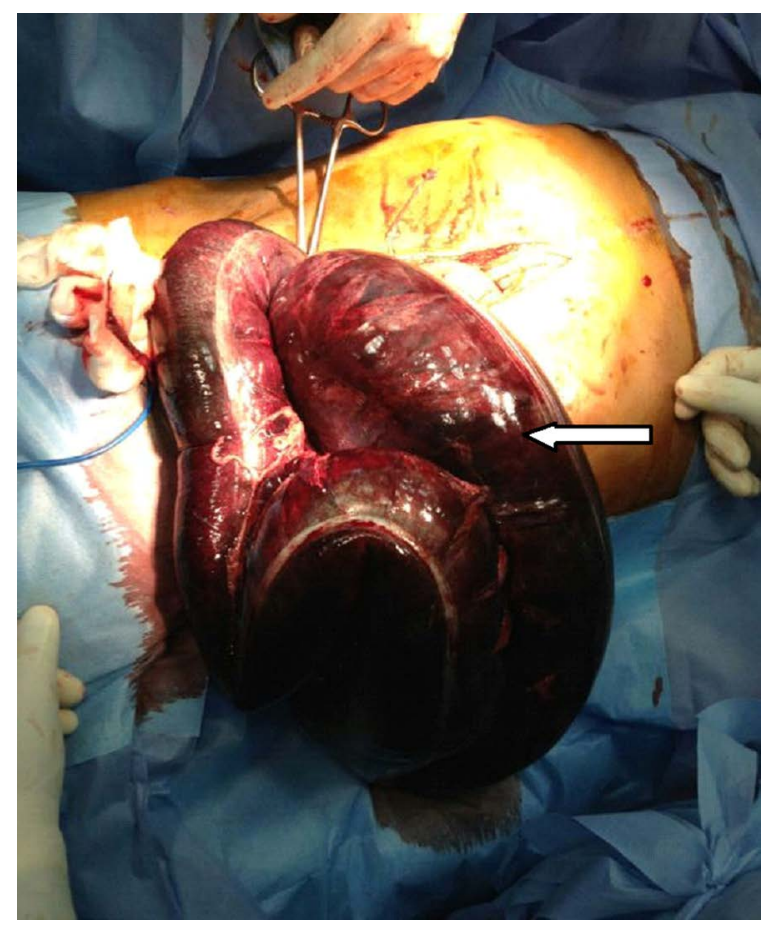

Figure 9. Intra-operative photograph showing dilated, twisted and gangrenous sigmoid flexure (white arrow).

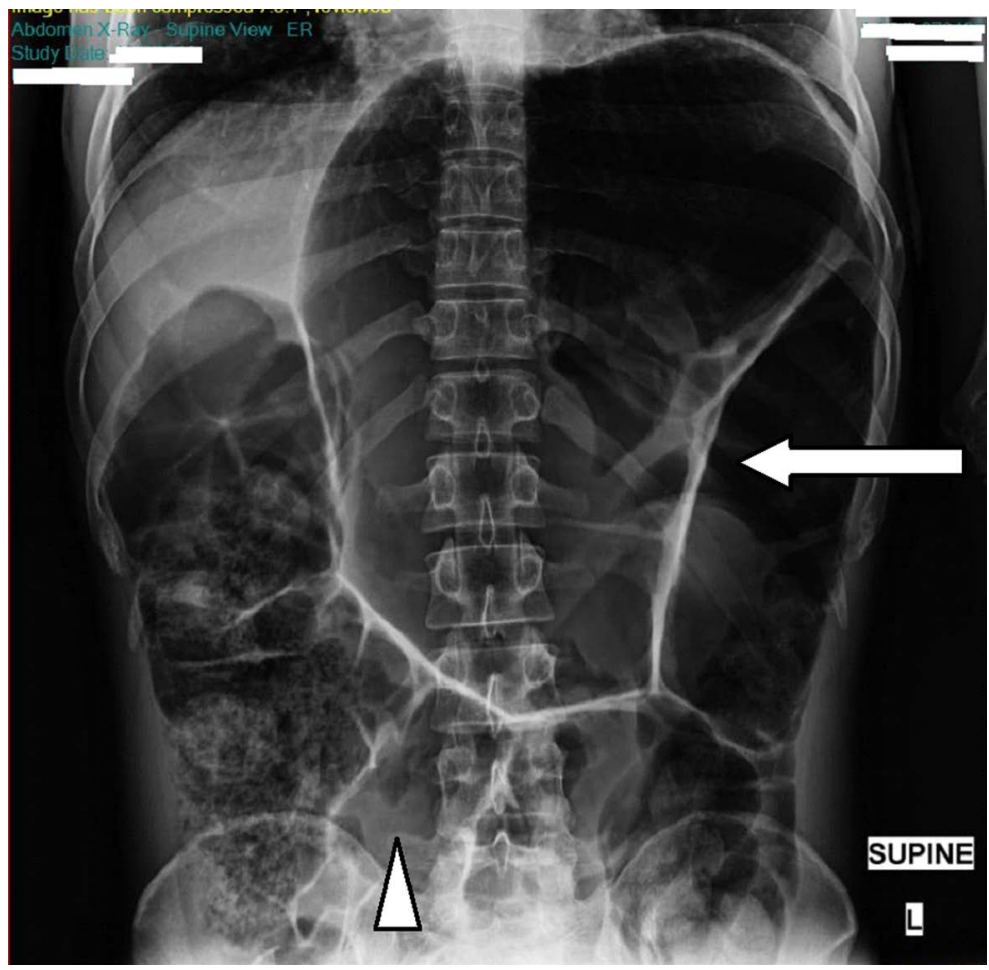

Figure 10. Plain supine abdominal radiograph shows twisted loop of bowel in splenic region (white arrow) with dilated large bowel loops (arrowhead).

Intraoperatively, the splenic flexure was found to be viable, dilated and twisted at its base. Proximal colonic dilatation was observed (Figure 14). The splenic flexure was resected and anastomosis performed. The patient's 


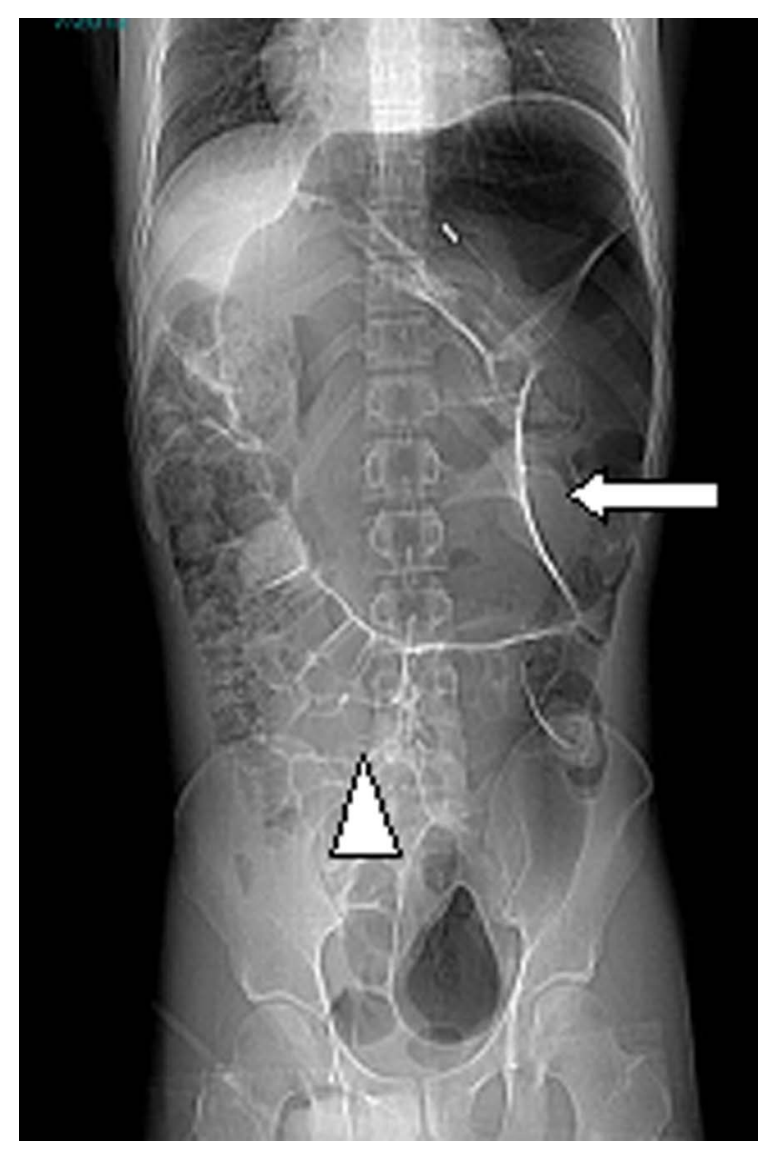

Figure 11. Scanogram of MDCT scan showing dilated sigmoid colon (white arrow) with dilated large bowel loops (arrowhead).

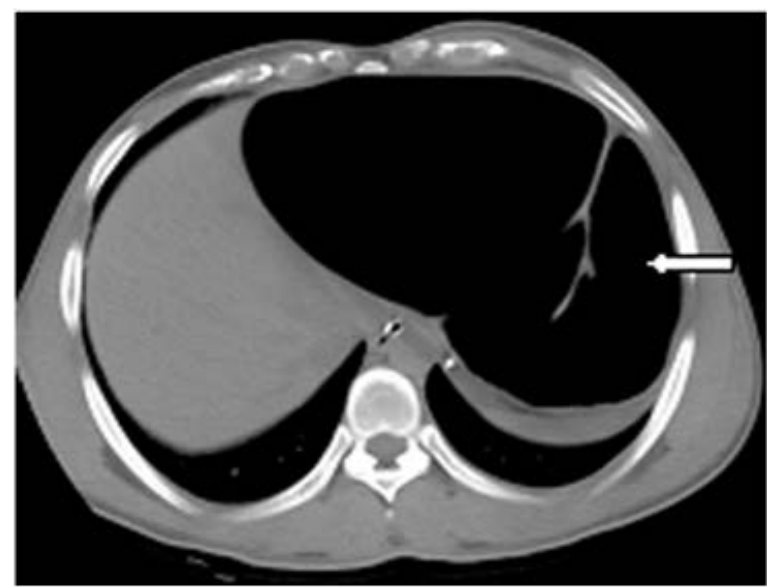

Figure 12. Axial image of Plain MDCT scan of abdomen showing dilated splenic flexure (white arrow).

recovery was uneventful.

\section{Discussion}

E. Parker described the first case of ileosigmoid knot in literature in 1845 [1], and the first Asian case was re- 


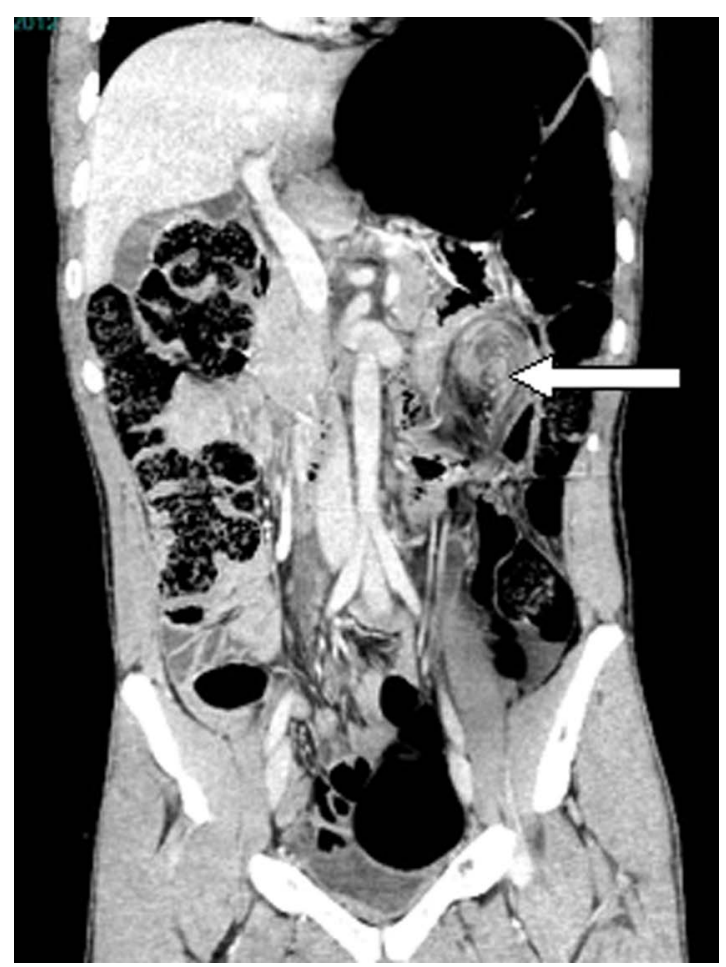

(a)

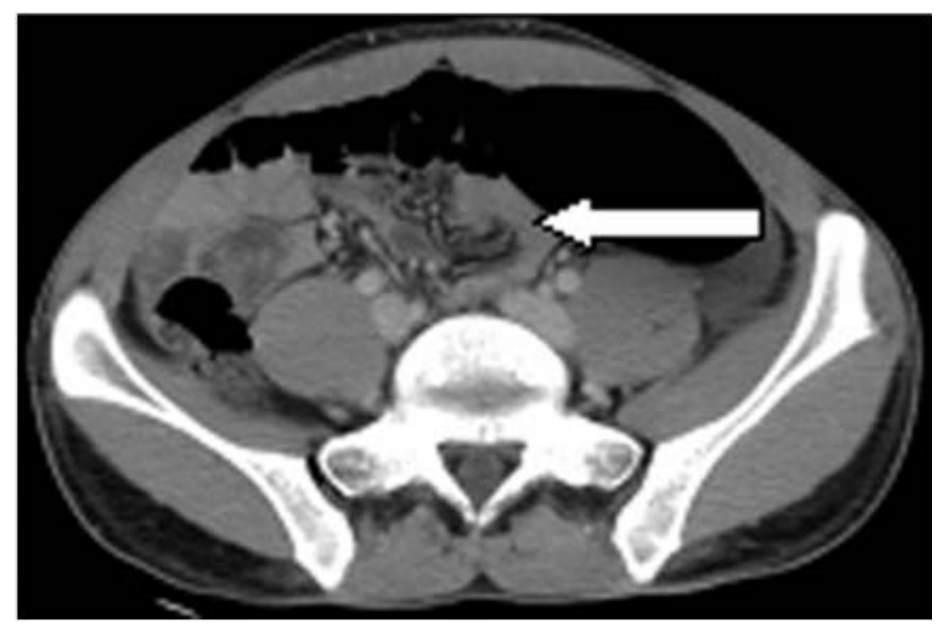

(b)

Figure 13. (a) (b) Coronal section and axial section of contrast enhanced CT scan of abdomen showing whirl sign (white arrows) due to twisted splenic flexure.

ported in 1940 by Shepherd, J.J. [2]. This condition is more common in males of African, Asian and Middle Eastern descent than in Caucasians [3]. Very few cases of this condition have been described in literature. This condition can be complicated by rapid progression to gangrene of both the ileum and the sigmoid colon, and therefore may be considered a surgical emergency [2]-[4]. Owing to the rarity of this condition, and the resulting lack of general awareness of it, preoperative diagnosis is difficult and likely often missed. MDCT however, has proved to be helpful in its early diagnosis.

The clinical presentation of ileosigmoid knot is quite non-specific and varies widely, ranging from abdominal pain and distention, to rectal bleeding. Clinical features of small bowel obstruction, imaging features of large bowel obstruction, and inability to pass a sigmoidoscope form a triad of diagnostic features for ileo-sigmoid 
knot [5]. Ileosigmoid knots can be classified into four categories, based on the relationship of the knotted loops to each other. In type I, the ileum twists around the sigmoid volvulus, which is seen in our case (Figure 15). In type II, the sigmoid colon twists around the ileum and in type III, the ileocaecal junction twists around the sigmoid colon. Cases in which the involved segment cannot be identified accurately fall under type IV [2] [4]. Current literature states that type I is most common, accounting for $53.9 \%-57.5 \%$ of cases, with type II and III being next in line (accounting for $18.9 \%-20.6 \%$ and $1.5 \%$ of cases respectively) [4] [6] [7]. The mean mortality rate is $6.8 \%-8 \%$ in non-gangrenous and $20 \%-100 \%$ in gangrenous cases. The presence of advanced age, associated medical problems, shock, bowel gangrene or perforation increases the mortality rate. Most radiologists describe the signs as inconstant or atypical and clinicians feel that the clinical signs are difficult to define preoperatively [2]-[4] [6]-[10].

Sigmoid volvulus is a condition in which sigmoid colon twists around its mesocolon (Figure 16). It is a common cause of large bowel obstruction after neoplasm and diverticulitis. Caecal volvulus is its close mimic, and differentiation between the two is possible by observing the absence of haustra in sigmoid volvulus com-

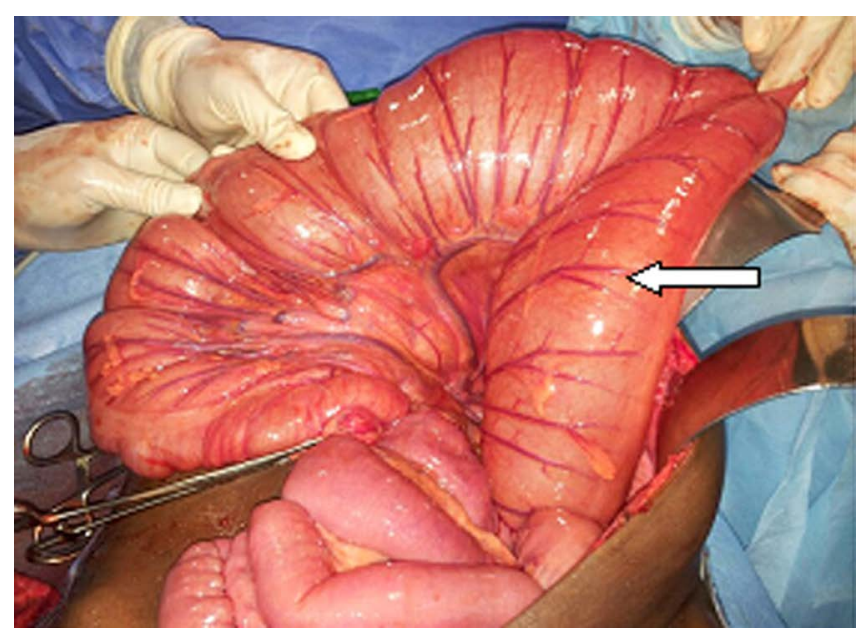

Figure 14. Intra-operative photograph showing dilated and twisted splenic flexure (white arrow).

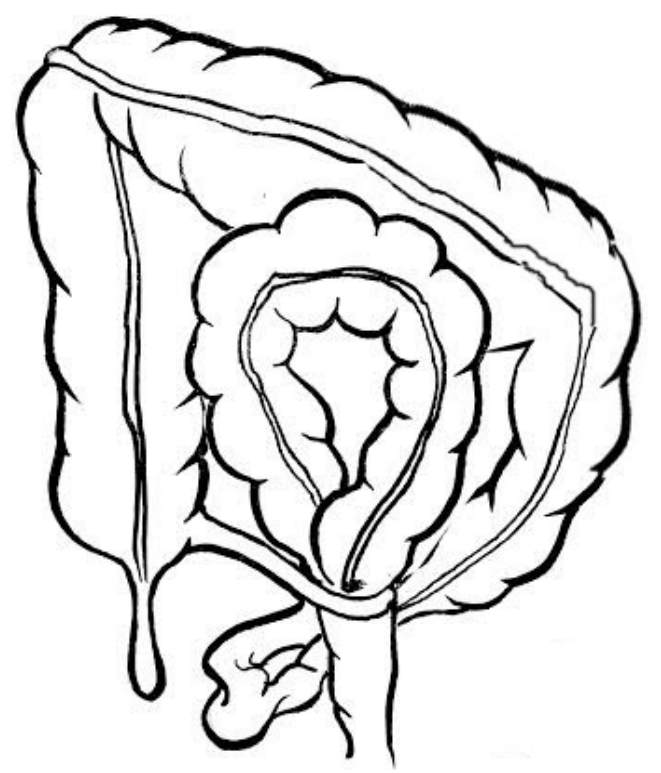

Figure 15. Line diagram showing dilated sigmoid colon with twisting of ileal loops at its base. Credit : Courtesy of Dr. Alexander Mylavarapu. 
pared to caecal volvulus. Also, sigmoid volvulus is directed toward the right upper quadrant, whereas caecal volvulus points to the left upper quadrant [11]. Many signs have been described in literature for sigmoid volvulus, including those seen in our case-Y sign, omega or horseshoe sign, bird's beak sign, coffee bean sign, bent inner tube or ace of spades sign, left pelvic overlap or left flank overlap sign, liver overlap sign, northern exposure sign, the whirl sign, Frimann-Dahl's sign and empty left iliac fossa sign [12]-[19].

Splenic flexure volvulus is an important and rare cause of large bowel obstruction. The splenic flexure is held in position by the phrenicocolic, gastrocolic, and splenocolic ligaments. These ligaments, along with the retroperitoneal location of the descending colon [20] prevent its movement. Mobility of the splenic flexure may be permitted in the setting of congenital absence of the supporting ligaments [21], or due to their surgical removal. A long mesentery causing redundancy of the splenic flexure may be another contributing factor [22]-[25]. The splenic flexure twists around its base and shows proximal colonic dilatation (Figure 17). The clinical features
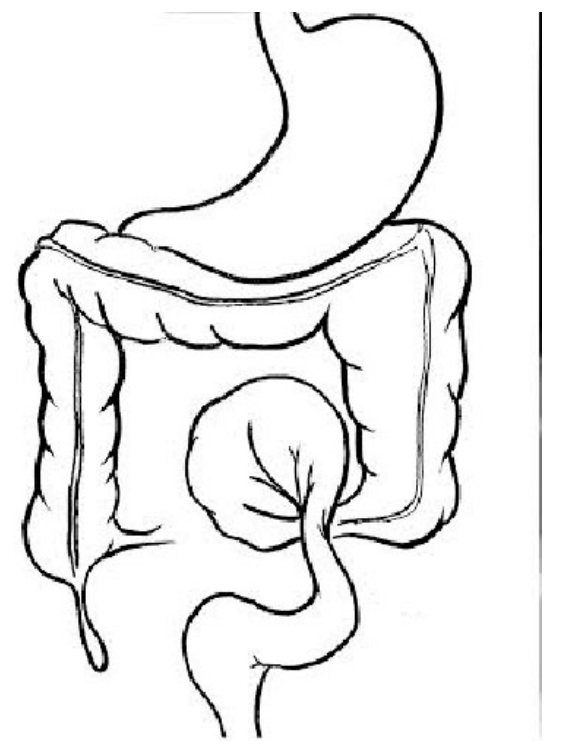

Figure 16. Line diagram showing twisting of sigmoid colon around its mesocolon. Credit: Courtesy of Dr. Alexander Mylavarapu.

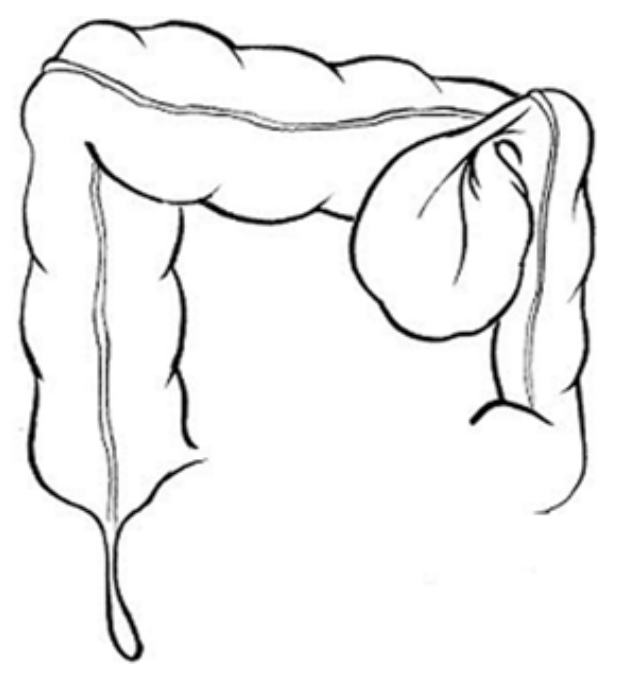

Figure 17. Line diagram showing twisting of splenic flexure with proximal bowel dilatation. Credit: Courtesy of Dr. Alexander Mylavarapu. 
are non specific. MDCT with intravenous contrast will demonstrate twisted splenic flexure of colon with dilated transverse and ascending colon as seen in the case diagnosed by us.

\section{Conclusion}

Volvuli is underdiagnosed surgical emergencies that have a significantly improved outcome with timely diagnosis and intervention. Given their non-specific clinical presentation, it becomes important to recognize the imaging features in time, thus reducing the chance of complications such as gangrene.

\section{References}

[1] Parker, E. (1845) Case of Intestinal Obstruction: Sigmoid Flexure Strangulated by the Ileum. American Journal of the Medical Sciences, 11, 464-465.

[2] Shepherd, J.J. (1967) Ninety Two Cases of Ileosigmoid Knotting in Uganda. British Journal of Surgery, 54, 561-566. http://dx.doi.org/10.1002/bjs.1800540615

[3] Alver, O., Oren, D., Tireli, M., Kayabasi, B. and Akdemis, D. (1993) Ileosigmoid Knotting in Turkey: Review of 68 Cases. Diseases of the Colon \& Rectum, 36, 1139-1147. http://dx.doi.org/10.1007/BF02052263

[4] Puthu, D., Rajan, N., Shenoy, G.M. and Pai, U.S. (1991) The Ileosigmoid Knot. Diseases of the Colon \& Rectum, 34, 161-166. http://dx.doi.org/10.1007/BF02049992

[5] Raveenthiran, V. (2001) The Ileosigmoid Knot: New Observation and Changing Trends. Diseases of the Colon \& Rectum, 44, 1196-1200. http://dx.doi.org/10.1007/BF02234644

[6] Atamanalp, S.S., Oren, D., Basoglu, M., Yildirgan, M.I., Balik, A.A., Polat, K.Y., et al. (2004) Ileosigmoid Knotting: Outcome in 63 Patients. Diseases of the Colon \& Rectum, 47, 906-910. http://dx.doi.org/10.1007/s10350-004-0528-9

[7] Atamanalp, S.S., Oren, D., Yildirgan, M.I., Bosoglu, M., Aydinli, B., Ozturk, G. and Salman, B. (2007) Ileosigmoid Knotting in Children: A Review of 9 Cases. World Journal of Surgery, 31, 31-35. http://dx.doi.org/10.1007/s00268-006-0255-6

[8] Akgun, Y. (1997) Management of Ileosigmoid Knotting. British Journal of Surgery, 84, 672-673. http://dx.doi.org/10.1002/bjs.1800840526

[9] Kakar, A. and Bhatnagar, B.N. (1981) Ileosigmoid Knotting: A Clinical Study of 11 Cases. Australian and New Zealand Journal of Surgery, 51, 456-458. http://dx.doi.org/10.1111/j.1445-2197.1981.tb05984.x

[10] Miller, B.J. and Borrowdale, R.C. (1992) Ileosigmoid Knotting: A Case Report and Review. Australian and New Zealand Journal of Surgery, 62, 402-404. http://dx.doi.org/10.1111/j.1445-2197.1992.tb07213.x

[11] Catalano, O. (1996) Computed Tomographic Appearance of Sigmoid Volvulus. Abdominal Imaging, 21, 314-317. http://dx.doi.org/10.1007/s002619900071

[12] Shaff, M.I., Himmelfarb, E., Sacks, G.A., et al. (1984) The Whirl Sign: A CT Finding in Volvulus of the Large Bowel. Journal of Computer Assisted Tomography, 8, 559-591.

[13] Peterson, C.M., Anderson, J.S., Hara, A.K., Carenza, J.W. and Menias, C.O. (2009) Volvulus of the Gastrointestinal Tract: Appearances at Multimodality Imaging. RadioGraphics, 29, 1281-1293. http://dx.doi.org/10.1148/rg.295095011

[14] Burrell, H.C., Baker, D.M., Wardrop, P. and Evans, A.J. (1994) Significant Plain Film Findings in Sigmoid Volvulus. Clinical Radiology, 49, 317-319. http://dx.doi.org/10.1016/S0009-9260(05)81795-7

[15] Frank, A.J., Goffner, L.B., Fruauff, A.A., et al. (1993) Cecal volvulus: the CT whirl sign. Abdominal Imaging, 18, 288-289. http://dx.doi.org/10.1007/BF00198126

[16] Balthazar, E.J., Birnbaum, B.A., Megibow, A.J., et al. (1992) Closed-Loop and Strangulating Intestinal Obstruction: CT Signs. Radiology, 185, 769-775.

[17] Hirao, K., Kikawada, M., Hanyu, H., et al. (2006) Sigmoid Volvulus Showing “a Whirl Sign” on CT. Internal Medicine, 45, 331-332. http://dx.doi.org/10.2169/internalmedicine.45.1643

[18] Javors, B.R., Baker, S.R. and Miller, J.A. (1999) The Northern Exposure Sign: A Newly Described Finding in Sigmoid Volvulus. AJR, 173, 571-574. http://dx.doi.org/10.2214/ajr.173.3.10470881

[19] Raveenthiran, V. (2000) Emptiness of the Left Iliac Fossa: A New Clinical Sign of Sigmoid Volvulus. Postgraduate Medical Journal, 76, 638-641. http://dx.doi.org/10.1136/pmj.76.900.638

[20] Jones, O., Srivastava, V. and Cochrane, R. (2010) Splenic Flexure Volvulus. BMJ Case Reports. http://dx.doi.org/10.1136/bcr.10.2009.2357

[21] Sachidanathan, C.K., Soehner, B. (1972) Volvulus of the Splenic Flexure of the Colon, Report of a Case and Review of 
the Literature. Diseases of the Colon \& Rectum, 24, 466-469. http://dx.doi.org/10.1007/BF02642659

[22] Blumberg, N.A. (1958) Volvulus of the Splenic Flexure of the Colon: Report of a Case with a Review of the Literature. British Journal of Surgery, 46, 292-295. http://dx.doi.org/10.1002/bjs.18004619720

[23] McGarity, W.C., Bobo, W. and Haynes, C.D. (1966) Volvulus of the Splenic Flexure of the Colon: Report of Two Cases and Literature Review. American Surgery, 32, 425-430.

[24] Poppel, M.H., Zeitel, B.E. and Abrams, R.M. (1956) Volvulus of the Splenic Flexure. American Journal of Digestive Diseases, 1, 380-386. http://dx.doi.org/10.1007/BF02233468

[25] Wray Jr., R.C. and Wangensteen, S.L. (1971) Volvulus of the Splenic Flexure and Cecum. Surgery, 27, 351-352. 